\title{
A proposal for the process of accreditation by portfolio for a GPwSI in respiratory medicine
}

\author{
Kevin Gruffydd-Jones*
}

\section{on behalf of a Working Group representing: General Practice Airways Group, British Thoracic Society, Primary Care Group Royal College of Physicians, Royal College of Nursing, National Respiratory Training Centre, Respiratory Education and Training Centres}

Box Surgery, Box, Wiltshire, SN14 8NA, UK

Received 14 June 2004; accepted 18 September 2004

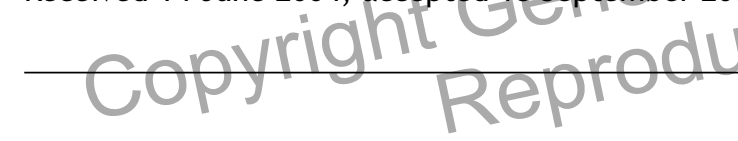

Introduction

The NHS plan proposes up to 1000 general practitioners with a special interest (GPwSIs) [1]. The rationale for employing GPWSIs in respiratory disease is:

(a) Government policy has encouraged a shift in emphasis in the provision of health care services away from the hospital to the community. Primary care organisations(PCOs) have a responsibility for commissioning and delivery of these services.

(b) Respiratory disease carries a huge burden for patients, society and the health service. Much of this burden falls upon primary care [2].

\footnotetext{
* Corresponding author. Tel.: +44 1225 742361; fax: +44 1225742646 .

E-mail address: Kevin.Gruffydd-Jones@gp-J83013.nhs.uk.
}

(c) Waiting lists for secondary care outpatient respiratory services remain long with a high number of admissions and in-patient days taken up by people with respiratory disease [3]

GPwSIs, working within PCOs, have the potential to provide improvements in all these areas by providing and organising community-based respiratory services [4].

The General Practice Airways Group (GPIAG) in conjunction with the Royal College of General Practitioners (RCGP) and in consultation with other respiratory-interested organisations has drawn up a framework of core activities and competencies for a GPWSI in respiratory medicine [5]. This emphasises that the GPwSI is not a glorified clinical assistant, but is a practising general practitioner with a special expertise in respiratory medicine. The key roles of the GPwSI are:

- Service development and leadership within a primary care organisation 
- Provision of intermediate clinical care in conjunction with local secondary care providers

- Education of and liaison with other health professionals within a primary care setting.

The framework document specifies the need for a process of accreditation for GPwSIs in respiratory medicine, but does not go into detail about how this process might take place. To this end the Educational Sub-committee of the GPIAG drew up an accreditation discussion document in 2003 [6]. This has been further discussed by a working party drawn from respiratory professional bodies within primary and secondary care, leading to the formulation of this current proposal document.

This document aims to set national standards for the accreditation of GPwSIs in respiratory medicine and is intended to be a useful guide for PCOs and GPwSIs alike. It could also act as a basis for devising standards of accreditation for GPwSIs by regulatory bodies within the Department of Health or General Medical Council.

\section{Aims of accreditation}

The aim of accreditation of a GPwSI in respiratory medicine is to ascertain that the practitioner has the necessary experience, knowledge, intellectuah and practical skills to fulfil competently the key roles outlined by the framework document (see above). In addition the GPwSI should continue to work as a generalist primary care practitioner. It is recognised that a GPWSI may vary in the extent to which all these functions are given priority according to local service requirements but the process of accreditation should encompass all these areas as a core national standard.

\section{Evidence of training for and successful acquisition of the required competencies}

\section{Generalist competencies}

PCOs will need to ensure that the GP is a competent and experienced generalist. Evidence of this can be provided by the system of re-accreditation proposed by the General Medical Council, but in the interim, evidence of generalist experience could include:

a. Membership of the Royal College of General Practitioners by examination b. Successful completion of summative assessment as defined by the Joint Committee of training for General Practitioners and

c. A current generalist professional portfolio indicating an active generalist education.

\section{Specialist competencies}

The specialist competencies of a GPwSI should be assessed by submission of a specialist professional portfolio to a local accreditation panel, constituted and working to national standards as outlined below.

\section{Core elements of the specialist portfolio}

(a) Track record of providing high quality respiratory care in line with national guidelines

This might involve presenting evidence of:

- Audit of respiratory work in practice

- Delivery of high quality respiratory services in a PCO/practice/nationally

- Specialist respiratory work in secondary care

- Active involvement in respiratory research and education

- Evidence of Working in a team to provide respiratory services

- Peer reviewed articles and research

(b) Personal development

Evidence of an active educational portfolio (reflective learning with learning outcomes) in respiratory medicine

(c) Analytical reflection on clinical events.

- Appraisal of 3 significant events

- Case history analysis detailing the decisionmaking rationale

(d) Submit a proposal for improving service delivery in a PCO (Max 2000 words)e.g. Submit a proposal for a GPwSI -led service in respiratory medicine in a $\mathrm{PCO} /$ this $\mathrm{PCO}$

- What are the issues?

- How do you make it happen?

- How do you evaluate success?

(e) Evidence of educational skills

Submit an outline for an educational event on a respiratory topic of your choice using Microsoft PowerPoint ${ }^{T M}$ with appropriate aims and learning outcomes.

(f) Assessment of practical skills (in adults and children)

- Taking an appropriate history and examination

- Ordering appropriate investigations and knowing their limitations 
- Appropriate secondary care referral

- Interpret and perform spirometry accurately

- Interpret ECG accurately

- Skin prick testing

- Recognition of the abnormal versus the normal chest X-ray

A more detailed list of the skills that could be tested is outlined in Appendix A.

It is proposed that these skills are assessed during supervised consultations (with an adult and paediatric respiratory consultant) of new patients in hospital outpatients (e.g. 12 diverse patients in 3 and 4 sessions per year). It is recognised that this may not be a fully rigorous approach to testing these skills but it is a pragmatic solution to assessing skills in the context of an educational portfolio rather than an exam setting.

\section{Role of a diploma in primary care respiratory medicine}

It is acknowledged that sections $b-f$ of the portfolio, outlined above, could be more rigorously tested by successful completion of a Diploma in Primary Care Respiratory Medicine.

Such a Diploma should teach and test the three core areas of competencies and knowledge required by the GPWSI:

- Specialist clinical skills and knowledge

- Service development and leadership skills

- Educational skills

Generic modules (applicable to GPwSls in other disease areas) could be developed to cover the training and assessment of service development/leadership and educational skills. The content of such modules is beyond the scope of this article but national standards could be set by generalist primary care bodies such as the Royal College of General Practitioners.

However, Appendix A outlines the possible specialist clinical roles of a GPwSI in respiratory medicine, together with the knowledge and competencies required to fulfil these roles.

At present no Diploma of Primary Care Respiratory Medicine exists which conforms to these proposals. It is hoped that by 2009 such a Diploma will form the core element of an accreditation portfolio. In the interim the process outlined in Section 4 is proposed as the method of accreditation.

\section{Membership of the accreditation panel}

- Secondary care adult respiratory specialist

- Secondary care paediatrician

- Expert Patient nominated by Asthma UK/BLF

- A PCO manager/health commissioner

- GPwSI nominated by the GPIAG

- Nominee of the Postgraduate Deanery

\section{Role of the accreditation panel}

The panel should provide initial accreditation and re-accreditation of the GPwSI. It is not the role of the accreditation panel to be involved in appraisal and mentoring.

\section{Register of accredited GPwSI's}

It is recommended that the General Practice Airways Group (GPIAG) hold a register of accredited GPwSIs in Respiratory Medicine and it is the role of the accredited GPwS to inform the Secretariat of the GPIAG (info@gpiag.org) that accreditation and re-accreditation has taken place.

\section{Timing of accreditation}

Once the system has been established for several years it is envisaged that GPwSls will only be appointed to specialist posts once they have been accredited. In the interim period an option will be to appoint a "GPwSI in training" who could work in the role of a GPwSI, for a year or two, at reduced remuneration, but with added paid time for training towards the requirements of accreditation.

\section{Payment for accreditation}

This should cover the professional time of the accreditation panel and administrative support. The fee would be the responsibility of the GPwSI, but it would be envisaged that an employing PCO would pay this as part of a GPwSI contract.

\section{Re-accreditation}

This should occur every 3 years using the generalist and specialist professional portfolios as key evidence of competencies 
Conflicts of interest: No conflicts of interest declared.

\section{Acknowledgements}

Members of the Working Party: Kevin GruffyddJones, GPIAG; Dermot Ryan, GPIAG; Bronwen Wagstaff, GPIAG; Richard Russell, British Thoracic Society; Monica Fletcher, National Respiratory Training Centre; Jane Scullion, Royal College of Nurses; David Bellamy, Royal College of Physicians; Jill Whatling, Respiratory Education and Training Centres. Additional thanks to the GPIAG Educational Sub-commitee (Malcolm Campbell, Hilary Pinnock, Philip Cotton) and to Sian Williams for her help with proof reading.

\section{Appendix A}

The possible specialist roles and clinical knowledge and skills of a General Practitioner with a Special Interest in Respiratory Medicine(GPwSI)

\section{ASTHMA}

Possible roles:

1. Facilitate the setting up of accurate asthma dis ease registers based on objective diagnoses, and with standardised, auditable data (e.g. Read Codes)

2. Set standards for auditable outcome measures e.g. percentage of asthmatics with an objective diagnosis of asthma

3. Raise standards in chronic asthma management

4. Possible outcome measures could include:

(a) Reducing asthma exacerbations

(b) Improving health status

(c) Use of self-management plans

5. Raise standard of acute asthma management, including out-of-hours services and developing integrated pathways of care with secondary care.

6. Outcome measures could include provision and use of oxygen, appropriate use of oral steroids, readmission rates etc.

7. Advising on asthma policy within schools.

Knowledge required:

Guidelines

- The GPwSI will be required to have a sound knowledge of National Guidelines for the Management of Asthma. e.g. SIGN/BTS Guidelines 2003 and NICE Guidance.

\section{Epidemiology}

- Changing prevalence and incidence of asthma and possible causes - local and national

- Impact of asthma in terms of morbidity and mortality

- Economic impact of asthma

- Natural history of disease

Pathology

- Cellular mechanisms

- Pathophysiology

Diagnosis

- Diagnostic criteria for asthma for all ages

- Differential diagnosis

- Objective tests

- A working knowledge of the use and interpretation of spirometry and peak flow measurements

- A basic knowledge of the role of predominantly secondary care and research investigations e.g. methacholine/histamine challenge tests, exhaled nitric oxide measurements

- Diagnostic criteria for exercise-induced and occupational'assthma

Non-pharmacological management Identification of pertinent trigger factors and role of allergen avoidance, environmental factors (especially smoking-see below under COPD), complementary therapy, exercise, immunotherapy and food intolerance, occupational factors.

Pharmacological management

- Outcome measures including economic indicators.

- Knowledge of the role, cost, efficacy and safety of asthma drugs and delivery devices.

Understanding of range of asthma types

- Management of chronic asthma

- Management of acute asthma in primary care in detail and a basic understanding of the management of acute asthma in hospital

- Managing asthma in special situations: the brittle asthmatic, asthma in pregnancy, occupational asthma, salicylate-sensitive asthma

- Exercise-induced asthma

- Managing asthma in schools

Organisation of care

- Routine asthma care in practice e.g. asthma templates, self-management plans, methods of 
structured review, identification of high-risk patients

- Influenza vaccination

- Relation of quality markers to GMS Contract

Managing the primary-secondary care interface

- Indications for referral to secondary care

- Shared care of chronic patients

- Management of acute exacerbations

Organisation of an asthma service in a PCO

- Audit and measuring quality.

The patient role

- Including awareness of role of Asthma UK

Skills required:

1. Ability to carry out and interpret spirometry

2. Ability to take and teach peak flow measurements

3. Ability to interpret chest X-rays

4. Ability to use and teach the use of inhaler devices

5. Ability to perform skin prick testing

6. Ability to manage the adult and child with acute asthma.

\section{COPD}

Possible roles:

1. Facilitate the setting up of an accurate COPD register across the PCO. This will involve help with spirometric diagnosis of COPD ranging from a practice-based approach to a central PCO-based intermediate care service.

2. Provide clinical advice on diagnostic and management uncertainties

3. Advice on chronic disease management within the practice

4. Development of community-based pulmonary rehabilitation

5. Co-ordination of preventative services e.g. smoking cessation services, immunisation with influenza and/or pneumococcal vaccines

6. Developing integrated care pathways with secondary care for:

(a) Prevention, management and follow-up of acute exacerbations in the community and post-hospital

(b) Referral guidelines to secondary care

(c) Use of oxygen therapy

(d) Use of nebulisers

(e) Palliative care

(f) Use of intermediate care beds

(g) Pulmonary rehabilitation
Knowledge required:

Guidelines

- A working knowledge of national guidelines e.g. BTS/NICE/GOLD

Epidemiology

- Incidence, prevalence, morbidity, mortality, economic impact

- Natural history and risk factors

Pathology

- Cellular mechanisms

- Pathophysiology

- Systemic effects

Diagnosis

- Clinical features

- Differential diagnosis

- Detailed knowledge of use and interpretation of spirometry, including expiratory flow-volume loops, reversibility testing and types of spirometer

- Basic knowledge of secondary care tests e.g. gas transfertests, CT scanning, induced sputum

Interpretation of chest X-rays

Management

- Assessment

- Knowledge of outcome measures: lung function tests and assessments of disability and handicap including dyspnoea scales, walking tests and health status measurements

- Smoking Cessation: knowledge of NICE guidance

- Epidemiology of smoking

- Benefits of smoking cessation with special regard to respiratory disease

- Detailed knowledge of efficacy, cost and side effects of NRT and bupropion.

- Use of carbon monoxide monitoring

Oxygen therapy

- Indications for long-term and on-demand oxygen therapy

- Cost

- Methods of delivery, compliance \& complications Pulmonary rehabilitation

- Role of pulmonary rehabilitation, methods, choice of patients

- Setting up a service for a PCO

Pharmacological therapy 
- Knowledge of the role, efficacy, side-effects, cost of drugs and delivery devices used in COPD

- How to carry out a nebuliser trial

- Management of acute exacerbations

Primary care management

- Use of self-management plans

- When to admit to hospital

- Flu and pneumoccocal vaccination

- Psychological management

Basic knowledge of secondary care management Organisation of care

- Organisation of COPD care within a practice, developing practice protocols and templates

- Organisation of care in a PCO - choosing outcome measures, education, audit

- The primary/secondary care interface including models of care e.g. "hospital at home"

- Smoking cessation

- The role of the patient including Breathe Easy Clubs

Skills required:

1. Ability to carry out and demonstrate spirometry procedures including reversibility testing.

2. Ability to interpret normal and abnormat volume-time and expiratory flow-volume traces.

3. Ability to carry out ECG procedures and in terpret traces illustrating common pathologies found in general practice.

4. Ability to interpret chest $x$-rays illustrating common chest pathologies found in primary care.

5. Ability to take accurate history and carry out competent examination.

\section{ALLERGIC DISEASE OF THE RESPIRATORY TRACT (rhinitis, asthma and food allergy)}

Possible roles:

- Develop local guidelines for rhinitis management

- Organisation of a community-based allergy service eg skin-prick testing

\section{Knowledge required:} Guidelines

- A working knowledge of national/international guidelines e.g. ARIA, BSACI

Epidemiology

- Incidence and prevalence including co-morbidity with asthma

Risk factors
- Including food allergy/intolerance

Natural History

Occupational Disease

Pathophysiology

- Mechanisms

Diagnosis

- Persistent $v$ intermittent

- Clinical presentation

- Differential diagnosis

- Diagnostic tests: IgE and antibody tests, skin prick testing

Management

- Allergen avoidance measures

- Immunotherapy

- Drug therapy: efficacy, safety and cost of different treatment modalities

- Anaphylaxis including patient self-management

- Complementary therapies

Organisation of care

- Setting up an allergy service in the PCO/practice

- The primary-secondary care interface, when to refer

- Provision of an immunotherapy service

Role of the BAF and the Anaphylaxis Society

The patient perspective

- Patient organisations and self-management

Skills required:

1. Carrying out and interpretation of skin-prick testing.

2. If an immunotherapy service were being organised, the GPwSI would need to demonstrate a competence to carry out the procedure and an ability to deal with anaphylaxis

3. Use inhaler devices

4. Teaching use of epinephrine pen

5. Examination of nasal cavities

\section{RESPIRATORY INFECTION}

Possible roles:

1. Organise preventative services e.g. influenza immunisation

2. Develop guidelines for management of respiratory infection

3. Develop integrated care pathways with secondary care for the management of childhood and adult infections including the provision of intermediate-care beds 
4. Organise diagnostic services for TB in areas at high-risk in liaison with secondary care services

Knowledge required: Guidelines

- Working knowledge of National Guidelines e.g. BTS Guidelines for the management of community acquired pneumonia, TB

Epidemiology, pathophysiology, clinical presentation and diagnosis, differential diagnosis, management, indications for hospital admission of:

- Upper respiratory tract infection

- Bronchitis

- Community acquired pneumonia

- TB

- Childhood infections such as croup, bronchiolitis. epiglottitis

- Influenza.

Organisation of care

- Influenza immunisation

- Diagnostic services for TB

- Primary-secondary care interface: models of care e.g. intermediate care beds, role of respiratory liaison nurse

Skills required:

1. Interpretation of chest $x$-rays showing common respiratory pathologies

2. Carrying out and interpretation of Mantoux tests

\section{ACUTE RESPIRATORY DISEASE}

Possible roles:

1. Organisation of protocols for acute respiratory disease within PCO.

2. Education and training of health professionals dealing with patients with acute respiratory problems.

\section{Knowledge required:}

- Differential diagnosis of the adult and child presenting with acute breathlessness

- Primary care management of the acutely breathless patient with specific management of:

- Acute asthma

Acute exacerbation COPD

Pulmonary Embolus

Pneumothorax

Foreign body inhalation

Acute adult and childhood infection including bronchiolitis and croup

- Anaphylaxis
Skills required:

1. Assessment of the acutely breathless child and adult.

2. Interpretation of ECG.

3. Ability to distinguish normal from abnormal chest $x$-ray.

4. Basic and Advanced (Defibrillator) Resuscitation Skills (could be part of generalist portfolio).

5. Ability to remove trapped upper airway foreign body in child or adult.

\section{OTHER RESPIRATORY DISEASE}

Possible roles:

1. Organisation of shared care protocols for diseases traditionally secondary-care based e.g. lung cancer.

2. Liaison with secondary care and palliative care providers for palliative care of patients with advanced respiratory disease.

3. Provision of specialist community-based services e.g. occupational lung disease, respiratory physiology tests.

4. Provision of specialist clinical advice to primary care professionats on diagnosis and disease management.

\section{Knowledge required:}

- A basic knowledge of the epidemiology and secondary care management of the following:

- A working knowledge of the clinical presentation, differential diagnosis and primary care management of the following:

- Interstitial lung disease

- Lung cancer

- Occupational lung disease

- Bronchiectasis

- Pulmonary manifestations of systemic disease e.g. Sarcoid

- HIV infection

- Sleep apnoea

- Cystic fibrosis

- Palliative care management of advanced respiratory disease

Disease prevention

- Smoking cessation

Organisation of care

- Primary/secondary care interface e.g. shared care of patients with lung cancer

- Palliative care of patients with advanced respiratory disease

- The patient perspective: self-help organisations, industrial injury claims 


\section{References}

[1] Department of Health. The NHS Plan: a plan for investment, a plan for reform. London: The Stationery Office. 2002.

[2] Royal College of General Practitioners Research Unit. Weekly Returns Service (Annual Report) Birmingham: RCGP Research Unit 2000.

[3] British Thoracic Society. The burden of Lung Disease. London BTS 2001.
[4] Williams S, et al. General practitioners with a special clinical interest: a model for improving respiratory disease management. British Journal of General Practice 2002;52(483):838-44.

[5] General practitioners with a special interest in respiratory medicine. Report of a working party of the General Practice Airways group and Royal College of General Practitioners. Prim Care Resp Journal 2003;12(2):38-41.

[6] Gruffydd-Jones K. Accreditation and appraisal of the general practitioner with a special interest in respiratory medicine. Prim Care Resp Journal 2003;12(4):107.

Available online at www.sciencedirect.com

science@direct.

\section{Available online at http://www.thepcrj.com}

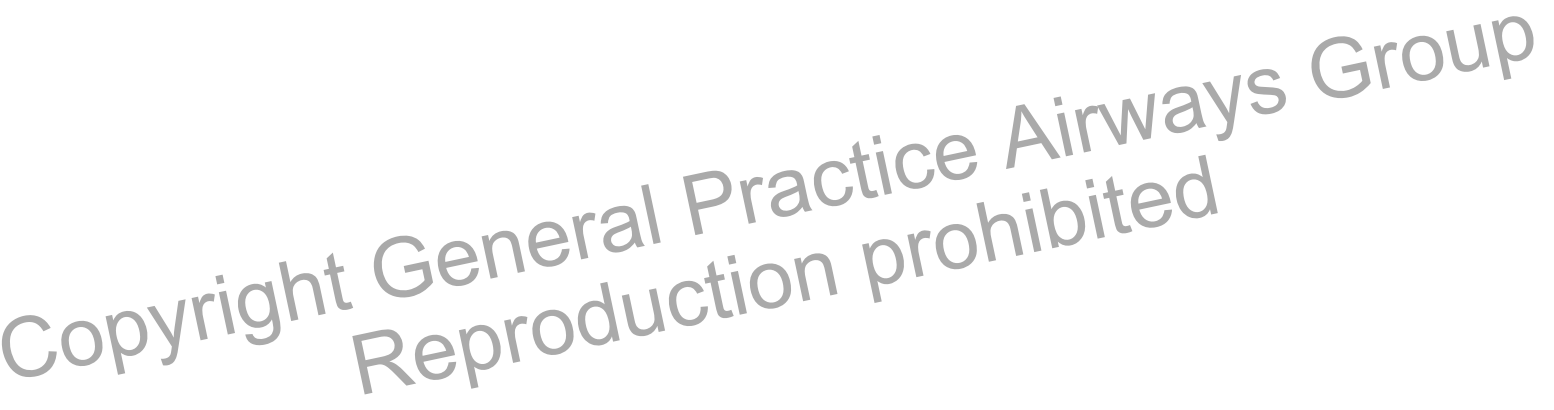

